\title{
La progression d'un objet partagé par une classe du primaire dans un environnement d'apprentissage en réseau en contexte d'éducation artistique
}

\author{
Esther Simard St-Pierre, Université TÉLUQ \\ Esther.SimardSt-Pierre@teluq.ca
}

Thérèse Laferrière, Université Laval

therese.Laferriere@,fse.ulaval.ca

Résumé : Cet article traite du travail d'une classe du primaire qui a utilisé un espace numérique de collaboration (Knowledge Forum) pour la création d'un objet d'art lors d'un projet intitulé De jeunes artistes sans frontières. Nous avons observé l'évolution de la création de cet objet en analysant le discours de la classe qui l'a réalisé. Nos résultats montrent qu'en interagissant entre eux à l'écrit ou par la création d'une variété d'artéfacts, les élèves se sont engagés dans une démarche qui leur a permis de faire progresser leurs interactions de manière à créer un objet partagé. Ils ont aussi également utilisé un langage artistique propre à leur culture.

Mots-clefs : Éducation artistique ; environnement d'apprentissage en réseau ; primaire ; objet partagé.

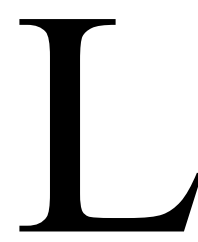

e déploiement d'Internet apporte des avancées remarquables dans tous les domaines et présente un fort potentiel éducatif. C'est le cas de l'École en réseau (ÉER), un modèle conçu, mis en œuvre et évalué par voie d'itérations successives grâce à une initiative destinée à favoriser l'égalité des chances pour les petites écoles de milieux éloignés, voire isolés du Québec. Né de la volonté du Ministère de l'Éducation, du Loisir et du Sport (MELS), ce projet de recherche-intervention avait le mandat de mettre en place des solutions aux difficultés vécues dans ces milieux, résultant notamment de baisses démographiques. Ainsi, dans ces milieux, les classes multiâges sont nombreuses, les enseignants sont trop souvent seuls dans leur établissement et les élèves ont peu de pairs du même âge ou de même niveau scolaire.

À ce jour, plusieurs écoles ont enrichi leur environnement d'apprentissage grâce au modèle ÉER (Laferrière, Hamel, Laberge et Allaire, 2005), caractérisé entre autres par l'accessibilité, tant pour l'enseignant que les élèves, à des personnes et à des ressources sur le Web et par des pratiques qui permettent notamment d'observer « la présence d'artéfacts d'apprentissage ou de création de connaissances, incluant des productions collectives » (Hamel, Turcotte et Laferrière, 2013). Afin que les enseignants et les élèves puissent interagir et vivre des activités en réseau, une infrastructure d'orientation et de soutien est à leur disposition sur une base quotidienne. Des partenariats entre différentes classes délocalisées sont mis en place et s'effectuent grâce à l'utilisation d'outils numériques et ce, en insistant sur le travail collaboratif et la coélaboration de connaissances (Laferrière et al., 2005 ; Scardamalia et Bereiter, 1994).

Les activités d'apprentissage ou de création de contenu dans l'ÉER sont cohérentes avec les attentes du Programme de formation de l'école québécoise et se réalisent dans toutes les matières en adoptant une approche multidisciplinaire. Toutefois, très peu d'activités concernent les arts. En dépit des politiques ministérielles québécoises qui font la promotion des arts dans le 
curriculum obligatoire, l'accès à l'éducation artistique est encore aujourd'hui un enjeu majeur et davantage dans les petites écoles de régions éloignées. L'enseignement des arts, tel que prescrit par le Programme de formation de l'école québécoise, reste un défi car il y a très peu de spécialistes des arts dans les écoles de niveau primaire. Il revient donc aux enseignants titulaires de réaliser les activités d'apprentissage dans ce domaine bien que ceux-ci n'aient habituellement que peu de formation en la matière.

Dans les écoles de l'ÉER, les activités d'apprentissage liées aux arts plastiques restent donc très limitées. Nous avions notamment constaté que, même si les élèves interagissent sur le Knowledge Forum $(\mathrm{KF})^{1}$ pour apprécier des œuvres d'art, ils ne s'engagent pas pour autant dans un processus de création complet. Le travail artistique leur demande de conceptualiser des idées et d'exprimer leur pensée certes, mais aussi de travailler de manière concrète avec la matière comme le soutiennent des chercheurs finlandais (Lahti,Seitamaa-Hakkarainen,Kangas, Härkkiet Hakkarainen, 2016 ; Seitamaa-Hakkarainen,Viilo et Hakkarainen, 2010). Dans un contexte de classe, travailler sur un objet développé collectivement peut devenir le point central de l'organisation générale des activités des élèves et se veut une excellente façon d'apprendre.

En cohérence avec les attentes du Programme de formation de l'école québécoise (PFÉQ), le volet intervention de notre démarche visait à délimiter un espace pour l'éducation artistique au sein d'une classe en réseau de l'ÉER pour mieux comprendre le rôle fondamental de la culture matérielle dans l'apprentissage appuyé par la technologie numérique en usage. La classe en réseau a été invitée à réaliser un objet partagé, ici une œuvre d'art. Ce travail a été effectué de manière simultanée et parallèle à un travail conceptuel de partage d'idées sur le KF et dans un travail plus " pratique » de mise en œuvre des idées en créant ou en utilisant des artéfacts matériels (plan, croquis et photos) lors d'ateliers. Le volet recherche de notre démarche s'articulait autour des questions suivantes :

1-Le discours produit par la classe en réseau permettait-il la création d'un objet partagé et, si oui, comment ? 2- De quelle manière la classe en réseau a-t-elle exprimé sa pensée artistique ?

\section{Cadre théorique}

Les communautés d'élaboration de connaissances (CoÉco) dans un environnement d'apprentissage en réseau s'adonnent à des activités de coélaboration de connaissances/création de connaissances ${ }^{2}$ (Laferrière et al., 2005). La création de connaissances est liée aux phénomènes émergents de la créativité collaborative dans les diverses théories de l'apprentissage soutenu par les technologies numériques. Elle fait référence à la troisième métaphore de l'apprentissage (Hakkarainen, Palonen,Paavolaet Lehtinen, 2004 ; Paavola, Lipponenet Hakkarainen, 2002 ; 2004) qui renvoie à celles proposées par Sfard (1998) ${ }^{3}$. Dans cette métaphore, les contextes et les acteurs impliqués suggèrent des motivations différentes pour chaque perspective ${ }^{4}$, mais se rejoignent là où un ensemble de pratiques sociales font avancer l'état des connaissances au sein d'une communauté (Paavola et al., 2004). Ce type de travail se produit lorsque les individus travaillent activement sur l'avancement et le développement d'un objet épistémique (Lakkala, Paavolaet Kosonen, 2009). Les objets épistémiques (ou artéfacts épistémiques) sont considérés comme des objets partagés par la communauté. Ils représentent un motif commun pour la communauté qui vise à faire progresser l'objet en convergeant et en canalisant les efforts de chacun des membres de la communauté vers le même objectif (Tan et Tan, 2014). Même lorsque l'objet partagé est conceptuel, comme la compréhension d'un sujet, l'amélioration des pratiques de travail, ou la conception d'un évènement ou d'un service, l'activité des individus devrait être organisée autour de la production d'objets matériels médiationnels, tels que des plans, des rapports ou des modèles 
visuels, qui aident concrètement à la médiation des efforts de collaboration (Lakkala et al., 2009; Seitamaa-Hakkarainen et al., 2010; Tan et Tan, 2014). Cette médiation est le processus de l'approfondissement d'un objet de connaissance par la création délibérée d'objets de connaissances et modifié progressivement par l'utilisation du discours (Härkki, SeitamaaHakkarainen et Hakkarainen, 2016 ; Hennessy et Murphy, 1999).

Dans une CoÉco d'élèves du primaire, les activités de création de connaissances impliquent davantage un travail conceptuel avec les idées où la communauté est engagée dans une démarche de résolution de problèmes. Les élèves sont alors encouragés à proposer des pistes de solutions et à élaborer des explications ainsi que des théories (Laferrière et al., 2005). Cela favorise le transfert de la responsabilité cognitive aux élèves (Bereiter, 2002; Bereiter et Scardamalia, 2014). Les questions et les problèmes de compréhension deviennent ainsi la force motrice du développement des connaissances (Scardamalia et Bereiter, 1994). Le questionnement des élèves exerce alors un rôle de médiation dans ce modèle en mettant en œuvre des cycles de création. Ce processus de résolution de problèmes est médié par des artéfacts de connaissances partagées, telles que des questions, des explications, des plans, des dessins, des diagrammes et des idées externalisées par l'écriture sur le KF, entre autres (Scardamalia et Bereiter, 1994).

Lorsque les élèves s'engagent dans un processus d'apprentissage à travers la création d'un objet matériel partagé, qui implique également un travail concret avec la matière (SeitamaaHakkarainen et al., 2010), ce travail peut devenir très similaire au processus de création des artistes professionnels. Selon Gosselin (2006), le travail de création artistique est une démarche de connaissance en soi. Effectivement, le motif des artistes qui s'engagent dans ce processus créatif est de transmettre une pensée et un savoir artistique. La création artistique est de plus en plus comprise comme étant construite là où se chevauchent les idées, les images et les savoirs : les idées sont ainsi incarnées dans une forme dite matérielle, ce qui repousse les limites d'une culture plutôt conceptuelle afin de la faire évoluer vers une culture matérielle de la création (Paavola et Hakkarainen, 2014).

\section{Méthodologie de recherche : \\ le codesign d'un projet d'art en réseau De jeunes artistes sans frontières}

De jeunes artistes sans frontières se veut être un projet permettant la concrétisation d'activités d'apprentissage en réseau en arts plastiques. Le devis réalisé est le fruit d'un travail conjoint entre deux étudiantes à la maîtrise en technologie éducative et deux enseignants titulaires au niveau primaire, l'un étant au Sénégal et l'autre au Québec. Cette collaboration (codesign) a donné lieu à la possibilité, pour les enseignants, de réfléchir et de concevoir leurs intentions pédagogiques en arts plastiques dans un environnement d'apprentissage en réseau. Ce travail se voulait une forme de développement professionnel (Voogt et al., 2015). Les enseignants, en collaboration avec les étudiantes et des artistes locaux, ont adapté des activités d'appréciation et de création artistique afin qu'elles puissent se vivre en réseau.

Le projet a pris forme dans deux environnements d'apprentissage en réseau : l'un au Québec, à Matapédia en Gaspésie et l'autre en Afrique subsaharienne, à Saint-Louis au Sénégal. Comme le démontre le tableau 1, le projet s'est échelonné sur plus d'une année scolaire (de la coélaboration des activités pédagogiques avec les enseignants à la coélaboration des objets d'arts par les élèves). Puisqu'il s'agit d'un projet en réseau, deux classes ont été impliquées dans un processus de collaboration et ce, avec des artistes de leur communauté. Le jumelage à un artiste local a permis aux élèves d'apprécier des œuvres liées à leur propre contexte culturel. Les élèves ont également visité des expositions et même l'atelier de l'artiste jumelé. De plus, des rencontres 
ponctuelles avec les classes et une séance d'échange avec les artistes ont été mises de l'avant par visioconférence.

Inspirées par le travail des artistes locaux, les classes ont coélaboré la proposition de création d'un objet d'art thématique, celui d'une sculpture représentant une embarcation : symbole de leur culture réciproque. Ce symbole culturel partagé faisait écho aux réalités des deux communautés en réseau, car toutes deux étaient desservies par d'importants cours d'eau et où la pêche avait un rôle de premier plan dans leur économie locale.

\section{Tableau 1}

Calendrier des activités en réseau

\begin{tabular}{|c|c|c|}
\hline $\begin{array}{l}\text { Étapes du } \\
\text { projet }\end{array}$ & Activités principales & $\begin{array}{l}\text { Description des activités et } \\
\text { supports à l'activité }\end{array}$ \\
\hline Printemps & $\begin{array}{l}\text { Recrutement des enseignants et } \\
\text { premières rencontres de planification } \\
\text { pédagogique. }\end{array}$ & $\begin{array}{l}\text { Coélaboration des activités de } \\
\text { création en réseau avec les } \\
\text { enseignants en visioconférence. }\end{array}$ \\
\hline Automne & Recrutement des artistes locaux. & $\begin{array}{l}\text { Coélaboration des activités } \\
\text { d'appréciation en réseau avec les } \\
\text { enseignants et les artistes en } \\
\text { visioconférence. }\end{array}$ \\
\hline Décembre & $\begin{array}{l}\text { Début des activités d'appréciation } \\
\text { avec les élèves en réseau. }\end{array}$ & Activités d'appréciation sur le KF. \\
\hline $\begin{array}{l}\text { Janvier- } \\
\text { Février }\end{array}$ & $\begin{array}{l}\text { Appréciation des œuvres des artistes } \\
\text { locaux. }\end{array}$ & $\begin{array}{l}\text { Visite de l'exposition et de l'atelier } \\
\text { de l'artiste local, rencontre des } \\
\text { artistes en visioconférence entre les } \\
\text { classes du Québec et du Sénégal. }\end{array}$ \\
\hline $\begin{array}{l}\text { Mars (début } \\
\text { de la } \\
\text { démarche de } \\
\text { création) }\end{array}$ & $\begin{array}{l}\text { Choix de la thématique de travail par } \\
\text { les élèves en réseau (objet de classe) } \\
\text { et création des équipes de travail } \\
\text { dans la classe du Québec (six } \\
\text { équipes de travail). }\end{array}$ & $\begin{array}{l}\text { Exploration de la thématique de } \\
\text { création en lien avec les deux } \\
\text { contextes (Québec-Sénégal) sur le } \\
\text { KF. } \\
\text { Coélaboration des premières idées } \\
\text { par croquis en atelier, mise en } \\
\text { commun et améliorations des } \\
\text { idées sur le KF. }\end{array}$ \\
\hline
\end{tabular}




\begin{tabular}{ll}
\hline $\begin{array}{l}\text { Avril } \\
\text { (démarche de }\end{array}$ & $\begin{array}{l}\text { Coélaboration de la création par la } \\
\text { classe } \\
\left.\text { création }^{5}\right)\end{array}$ \\
$\begin{array}{l}\text { (1 objet par équipe de travail, c'est-à- } \\
\text { dire six objets partagés ; objets 1 à }\end{array}$
\end{tabular}

6).
Élaboration des objets partagés (sculptures) en atelier et documentation du processus créatif et amélioration des idées sur le KF.

Exposition des œuvres réalisées dans chaque école. Activité d'appréciation des objets d'arts sur le KF par la classe en réseau.

Vernissage en réseau et retour sur l'expérience avec les deux classes et les artistes en visioconférence.

Les résultats présentés sont toutefois issus du travail effectué par la classe du Québec, 18 élèves du 3 e cycle du primaire (5e et 6 e année), et ne concernent que le volet création de l'objet d'art. Des données provenant du volet appréciation du corpus d'œuvres des artistes locaux font l'objet d'une analyse détaillée dans un mémoire de maîtrise (Simard St-Pierre, 2016). Également, un autre mémoire de maîtrise fait l'analyse des données provenant de la classe à St-Louis au Sénégal (Métivier, 2015).

L'ethnographie et l'analyse des artéfacts produits par la classe en réseau

La plateforme numérique permettant à la classe en réseau d'interagir a permis de conserver les écrits datés des élèves. Ainsi, nous avons pu suivre l'évolution de la création de l'objet partagé. Ce sont les différents artéfacts (écrits et visuels tels que les dessins et les photos des œuvres matérielles) provenant du discours qui ont fait l'objet d'une analyse. Les données recueillies dans le KF ont été extraites à l'aide de l'outil Séquences du contenu. ${ }^{6} \mathrm{~L}$ 'inclusion d'illustrations (dessins, photographies, images provenant du Web) a été réalisée manuellement, car le plugiciel n'en tenait pas compte. Enfin, l'outil Contributions de base sur le KF a permis d'extraire les données statistiques de l'activité de la classe en réseau.

Afin d'analyser la création de l'objet d'art, c'est la nature du discours ainsi que le type des artéfacts utilisés qui ont été analysés. L'analyse du discours a permis d'expliquer la nature des interactions de la classe à travers l'objet partagé et d'observer comment les élèves ont modifié ou non leur activité. Les composantes du discours supportant le processus de création (tableau 2) ont été analysées selon une grille de codage inspirée de Lahti et Seitamaa-Hakkarainen (2005) qui contenait les catégories suivantes : expériences de l'élève, rétroactions (commentaires-critiquesaméliorations), questions et idées. Nous avons adapté la grille au contexte de création artistique en ajustant chaque composante à la séquence des activités vécues par la classe en réseau (allersretours KF-atelier).

\section{Tableau 2}

Les composantes du discours de création 


\begin{tabular}{|c|c|c|}
\hline Composantes & Descriptions & Exemples \\
\hline $\begin{array}{l}\text { Sources d'inspiration/plans } \\
\text { de conception }\end{array}$ & $\begin{array}{l}\text { Idées ou influences qui animent } \\
\text { l'évolution de la création. }\end{array}$ & $\begin{array}{l}\text { Illustration/plan } \\
\text { illustré/croquis } \\
\text { «On pourrait utiliser les } \\
\text { saisons comme } \\
\text { thématique. » }\end{array}$ \\
\hline Processus & $\begin{array}{l}\begin{array}{l}\text { Énoncés réflexifs concernant le } \\
\text { travail pendant la phase de } \\
\text { création. }\end{array} \\
\text { Qui concerne les différentes } \\
\text { phases de la création et } \\
\text { l'avancement de l'œuvre. }\end{array}$ & $\begin{array}{l}\text { «Aujourd'hui, nous } \\
\text { avons eu beaucoup de } \\
\text { difficulté à peindre sur la } \\
\text { matière plastique. » }\end{array}$ \\
\hline $\begin{array}{l}\text { Expériences de } \\
\text { création/retour sur le } \\
\text { processus }\end{array}$ & $\begin{array}{l}\text { Énoncés réflexifs concernant le } \\
\text { résultat de sa propre création. } \\
\text { Retour sur l'expérience de } \\
\text { création. } \\
\text { Établit une synthèse de l'œuvre. }\end{array}$ & $\begin{array}{l}\text { « Notre sculpture est très } \\
\text { solide, maintenant. Elle } \\
\text { représente les différentes } \\
\text { saisons avec ses couleurs } \\
\text { variées. » }\end{array}$ \\
\hline $\begin{array}{l}\text { Expériences de } \\
\text { création/appréciations }\end{array}$ & $\begin{array}{l}\text { Énoncés réflexifs concernant le } \\
\text { résultat de la création des } \\
\text { pairs. }\end{array}$ & $\begin{array}{l}\text { «Votre dessin est très } \\
\text { réussi. Il y a beaucoup de } \\
\text { couleurs et c'est très } \\
\text { joyeux. » }\end{array}$ \\
\hline Rétroactions/questions & $\begin{array}{l}\text { Commentaires critiques pour } \\
\text { encourager ou remettre en } \\
\text { question le travail des pairs. } \\
\text { Qui permet l'amélioration de } \\
\text { certains énoncés. } \\
\text { Concerne les interactions entre } \\
\text { les différents groupes de travail } \\
\text { pour l'avancement de l'œuvre. }\end{array}$ & $\begin{array}{l}\text { «On vous suggère } \\
\text { d'utiliser du ruban } \\
\text { adhésif pour fixer la } \\
\text { base. » } \\
\text { « Je ne suis pas d'accord } \\
\text { avec ton idée, on pourrait } \\
\text { travailler avec de la pâte } \\
\text { à modeler.» } \\
\text { «Comment allez-vous } \\
\text { réaliser votre plan?» }\end{array}$ \\
\hline
\end{tabular}

De plus, puisqu'il s'agissait d'une démarche de création artistique, nous nous sommes interrogées sur la nature même de l'objet d'art produit par les élèves, soit les caractéristiques artistiques de l'objet d'art (voir tableau 3). Pour bien saisir la nature de l'objet, trois catégories ont été mises à contribution (esthétiques, matérielles et personnelles et expressives). Cette grille est une adaptation de celle de Lathi et Seitamaa-Hakkarainen (2005) qui intégrait une catégorie 
fonctionnelle relative à un objet de design. Nous l'avons ainsi remplacée par les catégories matérielles et personnelles, soit des catégories davantage orientées vers un objet d'art.

Concrètement, les qualités esthétiques sont liées ici à la recherche du beau (Souriau et Souriau, 2010), alors que les expressives démontrent les qualités symboliques et fonctionnelles de l'œuvre (Lamb et Kallal, 1992). Les considérations matérielles d'une œuvre se définissent par sa composition. Par exemple, les matériaux d'un tableau sont la toile et les pigments colorés que la peinture y étale. Le caractère personnel de l'objet d'art est davantage lié à la culture propre de l'individu à travers ses références sociales et émotionnelles (Vygotsky, 1971).

Selon Hakkarainen (2003), la présence de questions explicatives dans le discours des élèves semble être la résultante d'une activité de création de connaissances soutenue. Suivant cette assise théorique, nous avons attribué le code question à chaque phrase interrogative. Par la suite, nous avons attribué un code spécifique afin d'en déterminer l'auteur, soit élève ou enseignante.

\section{Tableau 3}

Grille des considérations artistiques de l'objet d'art (inspirée de Seitamaa-Hakkarainen et al., 2005)

\begin{tabular}{|c|c|c|}
\hline Qualités & Définitions & Exemples \\
\hline Esthétiques & $\begin{array}{l}\text { Qui témoignent de l'aspect } \\
\text { visuel et du langage plastique } \\
\text { (couleur, forme, grosseur, } \\
\text { etc.) }\end{array}$ & $\begin{array}{l}\text { «Mon bateau est beaucoup } \\
\text { coloré.» }\end{array}$ \\
\hline Matérielles & $\begin{array}{l}\text { Composition concrète de } \\
\text { l'œuvre en lien avec la } \\
\text { matière. }\end{array}$ & $\begin{array}{l}\text { «Je te donne l'idée de faire } \\
\text { les bois d'orignal en papier } \\
\text { d'aluminium.» }\end{array}$ \\
\hline Personnelles et expressives & $\begin{array}{l}\text { Éléments de culture, propres } \\
\text { à l'individu (référents } \\
\text { culturels et émotionnels) }\end{array}$ & $\begin{array}{l}\text { «Il y a présence d'un cheval } \\
\text { dans notre bateau. Le cheval } \\
\text { est important pour nous et } \\
\text { pour le Québec puisque c'est } \\
\text { le premier moyen de } \\
\text { transport.» }\end{array}$ \\
\hline
\end{tabular}

Également, les codes objet-1, objet-2, objet-3, objet-4, objet-5, objet-6 et objet-classe ont été attribués aux contributions des élèves sur le KF afin de cerner l'évolution de chaque objet partagé. L'objet-classe est associé à l'élaboration de la proposition de création thématique à laquelle l'ensemble de la classe en réseau a contribué. Par la suite, la classe en réseau s'est divisée en six sous-groupes de travail (équipes) afin de faire avancer les objets 1 à 6 .

\section{L'accord interjuge}

Le coefficient d'accord interjuge alpha de Krippendorff (2004)a été appliqué aux grilles présentées ci-dessus pour le codage des contributions des élèves (nature du discours de création et nature des considérations artistiques de l'objet d'art). Cet interjuge a mesuré la compréhension des 
différentes catégories d'une unité de sens : soit la totalité du contenu d'une note, soit une idée qui s'illustre par une phrase, un paragraphe ou un croquis (Chi, 1997). Deux analystes différents ont utilisé les grilles afin d'en assurer la fiabilité (inter-rater reliability) et pour vérifier la clarté des catégories. L'accord minimal entre les analystes pour chaque ensemble de codes est de .80. Les deux codeurs ont atteint un taux d'accord de 0.93 (nature création) et de 0,87 (considérations artistiques). La discussion a permis de résoudre les désaccords survenus lors du codage.

\section{Présentation des résultats}

La réalisation des différents objets d'art a généré 175 contributions avec une moyenne de 10,75 notes rédigées par auteur, contenant un écart-type de 3,69. Au total, l'enseignante a contribué à la hauteur de 31 notes écrites (c'est-à-dire $17 \%$ des contributions) tandis que $82 \%$ des contributions ont été attribuées aux élèves. Six sous-groupes de travail ont été constitués afin de faciliter le développement de l'objet d'art : ce sont donc six sculptures qui ont été réalisées.

\section{Figure 1}

Le pourcentage des composantes du discours de création par objet partagé

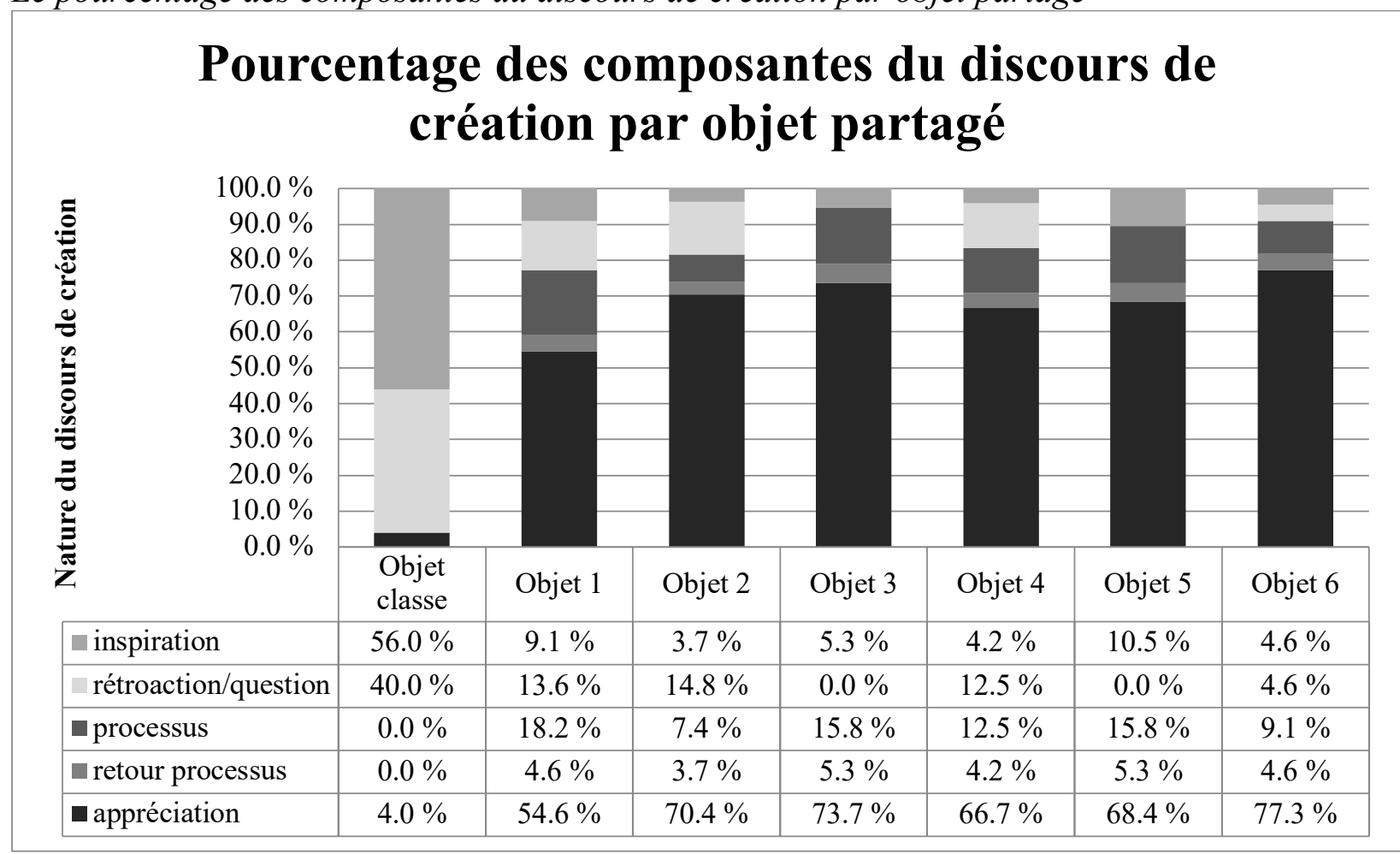

Note. Sur l'ensemble du discours produit par les élèves, ce sont $14 \%$ des contributions du discours de création (objet-classe $=56 \%$, objet-1 $=9,1 \%$; objet $-2=3,7 \%$; objet-3=5,3\%; objet$4=4,2 \%$; objet-5=10,5\%; objet- $6=4,6 \%$ ) qui ont été identifiées comme étant des sources d'inspiration/plans de conception. Pendant la création de l'objet matériel en atelier (composante du processus), les élèves ont partagé des énoncés réflexifs à la hauteur de $11 \%$ du discours de création ( $0 \%$ pour l'objet-classe, $18,2 \%$ pour l'objet-1 ; 7,4 \% pour l'objet-2 ; 15,8\% pour l'objet-3 ; 12,5\% pour l'objet-4 ; 15,8\% pour l'objet-5, 9,1\% pour 1'objet-6). $4 \%$ des 
contributions du discours de création (objet-classe $=0 \%$, objet- $1=4,6 \%$; objet- $2=3,7 \%$; objet$3=5,3 \%$; objet $-4=4,2 \%$; objet $-5=5,3 \%$; objet $-6=4,6 \%$ ) sont liées aux résultats de leur propre création (expériences de création et retour sur propre processus). L'essentiel du discours de création produit par la classe en réseau concerne l'expérience création/appréciations avec une nette majorité du discours : $58 \%$ répartis comme suit pour chaque objet : objet-classe $=4 \%$; objet- $1=54,6 \% ;$ objet $-2=70,4 \%$; objet $-3=73,7 \%$; objet $-4=66,7 \%$; objet $-5=68,4 \%$; objet $6=77,3 \%$.

Enfin, les rétroactions/questions représentent $13 \%$ (objet-classe $=40 \%$; objet- $1=13,6 \%$; objet $-2=14,8 \%$; objet $-3=0 \%$; objet $-4=12,5 \%$; objet $-5=0 \%$; objet $6=4,6 \%)$ du discours de création (voir tableau 4 pour des exemples de progression du discours). Dans cette catégorie, ce sont également 61 questions qui ont été posées ; 33 questions par les élèves (54\%) et 28 par l'enseignante $(45 \%)$.

Les artéfacts visuels, quant à eux, ont été utilisés pour appuyer essentiellement trois composantes du discours de création: sources inspiration/plans de conception, processus et expériences de création/retour sur le processus (voir tableau 4 pour quelques exemples). Six croquis des élèves ont été ajoutés au discours afin d'illustrer leur plan de conception (en moyenne 1 croquis par objet); 5 références visuelles provenant du Web ont été partagées afin d'appuyer leur source d'inspiration; 6 photos des œuvres ont été utilisées pour appuyer le processus de création (en moyenne une photo par objet) et six photos des œuvres pour illustrer le retour sur leur création (expériences de création/retours sur le processus). Nous avons énuméré 21 notes coauteurs ainsi que 23 artéfacts visuels (six croquis, 12 photos de l'œuvre en évolution et cinq illustrations provenant du Web) qui ont servi à l'élaboration des objets.

\section{Tableau 4}

Exemple de progression du discours concernant l'objet-1 sur le KF

Contributions des élèves $\begin{aligned} & \text { Codes } \\ & \begin{array}{l}\text { [nature du discours de } \\ \text { création > source } \\ \text { d'inspiration/plan de } \\ \text { conception] } \\ \text { [considérations } \\ \text { artistiques >personnelles } \\ \text { et expressives] }\end{array}\end{aligned}$

[Nouvelle idée] Notre idée est originale parce que notre bateau a des bâtons de hockey, une fleur de lys, un cheval avec des bois d'animaux, un saumon avec des bois et des personnes qui sourient. Ça nous représente parce qu'il y a la chasse avec les bois, la pêche avec le saumon, l'agriculture avec le cheval, le patrimoine avec le cheval également, le Québec avec la fleur de lys, la joie avec le multicolore et les têtes souriantes et le hockey avec les bâtons. 


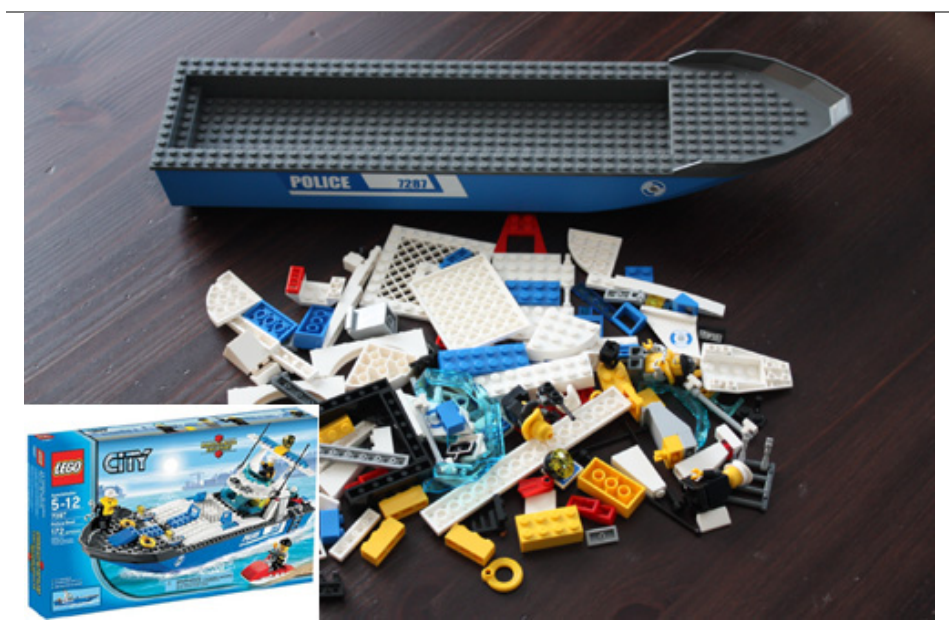

[nature du discours de

création $>$ source

d'inspiration/plan de

conception]

[considérations

artistiques

>personnelles et

expressives]

Voici notre source d'inspiration un canot LEGO.

[Explication] comment allez-vous faire les faces d'animaux sur le bout du bateau en LEGO ?

[nature du discours de création $>$ question]

[considérations

artistiques $>$ matérielles]

[Nouvelle information] On a demandé à [nom d'un élève] pour [nature du discours de qu'il fasse les têtes en pâte à modeler. création $>$ processus]

[considérations artistiques $>$ matérielles]

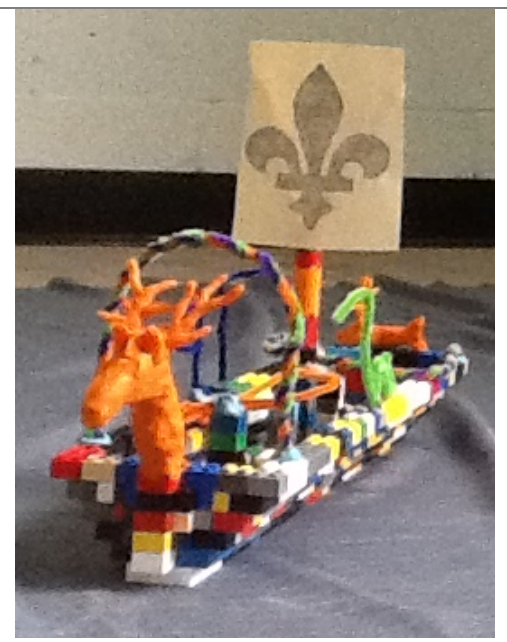

[nature du discours de création $>$ processus] [considérations artistiques $>$ matérielles]

Cette semaine, nous avons eu un pépin. Ce problème est la fragilité de notre structure. Nous n'avions pas prévu de mettre des curepipes. Nous allons aussi rajouter un bâton de hockey.

[Nouvelle information] [nature du discours de On vous propose de faire une plus grosse base et plus de gommette pour faire tenir le KD. création $>$ rétroaction] [considérations artistiques $>$ matérielles] 
[Nouvelle information] Pour que le signe de (KD) se tienne, vous pourriez le faire en LEGO aussi.

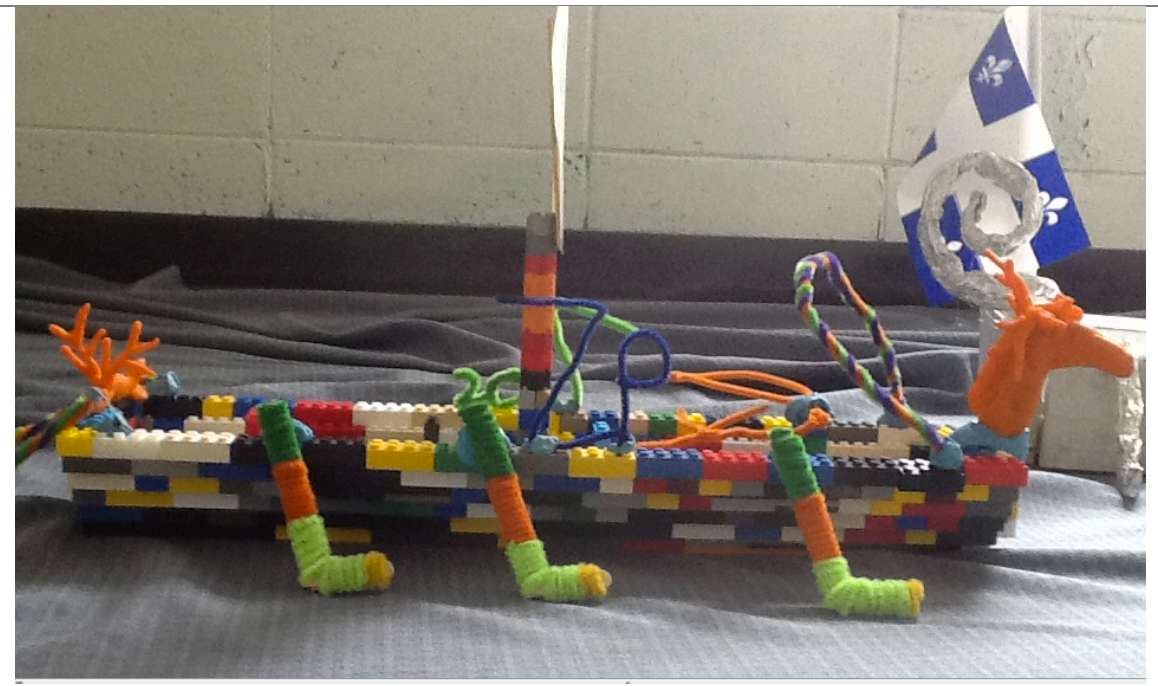

Notre bateau est original parce qu'il est très coloré, il apporte la joie et il a un drapeau. Notre bateau nous représente parce que nous aimons tous la chasse et la pêche. Les bâtons de hockey représentent [noms des co-équipiers]. Puisque nous venons tous du Québec, la fleur de lys et le cheval nous représentent bien. Nos initiales nous représentent bien aussi. Le cheval est important pour nous et le Québec puisque c'est le premier moyen de transport. [nature du discours de création $>$ rétroaction] [considérations artistiques $>$ matérielles]

Enfin, l'analyse du discours écrit a également permis d'identifier les caractéristiques artistiques des objets d'art (voir tableau 4 pour des exemples). D'après la figure 2, ces considérations varient grandement d'un objet à l'autre. Au total, ce sont 281 unités de sens qui ont été relevées du discours de création : soit une phrase, un paragraphe, le contenu total d'une note ou un artéfact visuel. Selon le total des contributions de la classe en réseau, ce sont $26 \%$ des considérations du discours qui concernent les qualités esthétiques de l'œuvre. Les considérations matérielles représentent $35 \%$ du discours de création. Enfin, 39\% du discours s'applique aux qualités personnelles et expressives. Aucune considération esthétique n'a donc été traitée lors de l'élaboration de l'objet-classe, tandis que pour l'objet-2, les considérations sont en grande majorité esthétiques. Pour sa part, l'objet-3 a fait état de considérations personnelles et expressives plus marquées que celles des autres équipes. Les considérations matérielles ont été davantage exprimées lors du développement de l'objet-1.

\section{Figure 2}

Les considérations esthétiques de l'objet d'art par objet partagé 


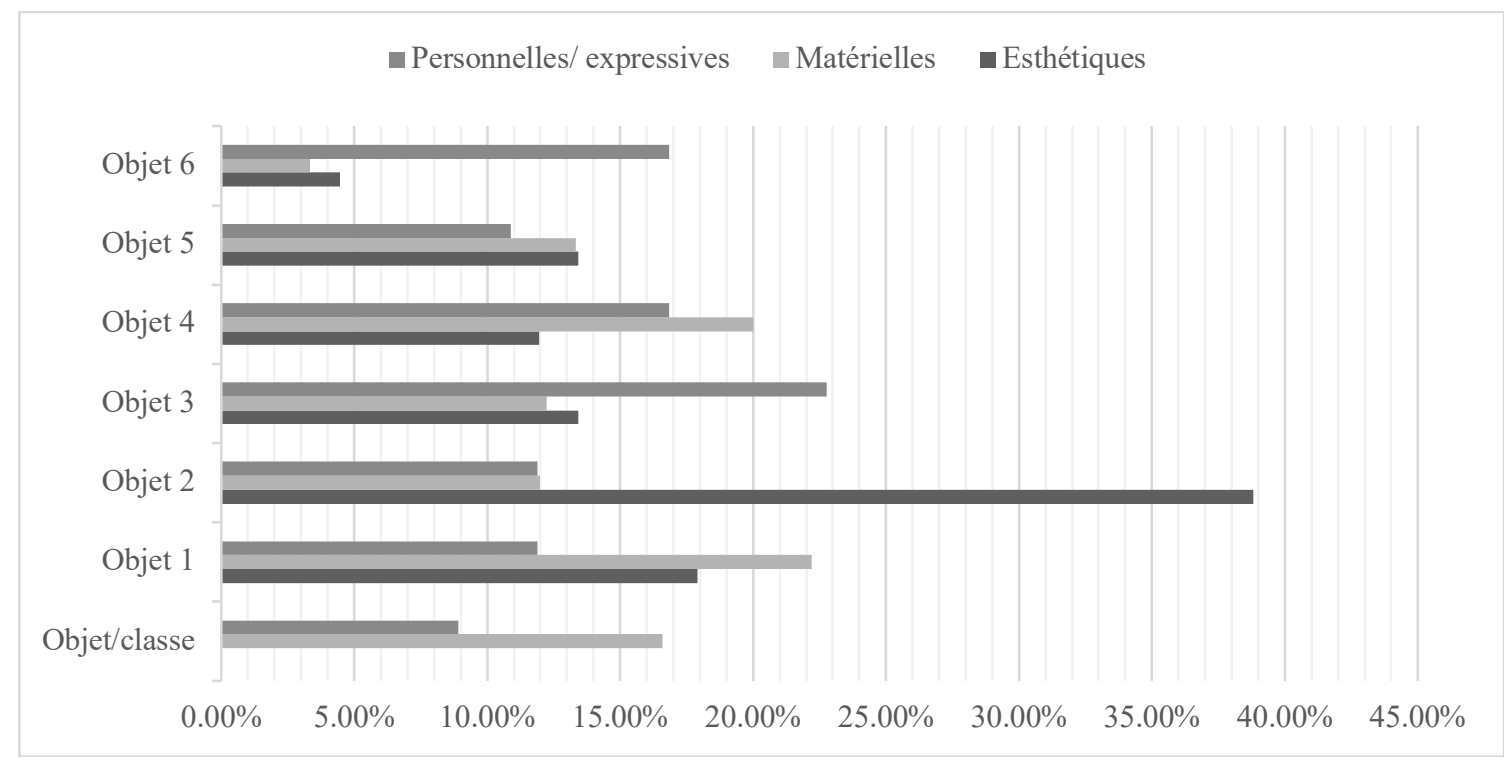

\section{Discussion à propos de la progression de l'objet d'art partagé}

L'objet d'art a été construit progressivement à partir des efforts de collaboration de toute la classe en réseau. Nous avons ainsi analysé la progression de l'activité de création partant du discours. Cette analyse a permis de comprendre la nature des interactions à travers l'objet partagé. De plus, considérant que selon Lakkala et al., (2010), l'activité d'une communauté doit être organisée autour de la création d'artefacts matériels qui permettent d'appuyer le travail sur l'objet partagé, l'activité de la classe en réseau a été organisée autour de la production d'objets matériels. Cette analyse nous a permis de comprendre que ces objets ont soutenu les efforts de collaboration des élèves afin de faire progresser l'objet d'art. Enfin, afin de cerner de quelle manière la classe en réseau a exprimé sa pensée artistique, nous avons relevé et analysé les différentes considérations artistiques du discours sur l'objet d'art. Ce qui suit constitue nos principaux constats.

\section{Une dynamique de classe favorisant les interactions à l'écrit}

Les pratiques de connaissances sur un objet partagé impliquent les élèves dans une dynamique qui génère la création d'un savoir collectif. Pour mettre en œuvre cette dynamique il faut encourager les interactions entre les élèves de la classe.

Dans le cadre de cette étude, les interactions associées à la composante rétroactions/questions sont demeurées timides. Les résultats montrent que les interactions plus soutenues ont été présentes lors de l'élaboration de l'objet-classe où les élèves devaient clairement définir les buts, les objectifs et les contraintes de l'objet d'art. Pendant la phase de création, le discours entre les différents sous-groupes de travail a permis d'apporter des propositions d'amélioration de leurs objets d'art, mais dans une proportion moindre. De plus, ce ne sont pas tous les objets qui ont progressé au moyen des interactions sur le KF comme l'illustre la figure 1. Dans une nette majorité, le discours produit par les élèves a permis le partage de leur expérience de création et de la synthèse de leur œuvre.

Par le questionnement, l'enseignante a également contribué à l'avancement du discours. Des questions d'encadrement du processus de création ont été énoncées à quelques reprises : «Aujourd'hui, nous débutons le projet de création en arts plastiques. Comment allons-nous faire pour rendre ce canot-embarcation de manière artistique, originale et personnelle? À la deuxième 
semaine du projet de création, l'enseignante relance les élèves en posant la question suivante : «Voici une semaine que le projet de création est commencé. Où en êtes-vous? Quelles améliorations avez-vous faites ? et «Nous voici vers la fin du projet de création, nous aimerions savoir où vous en êtes rendus sur la construction des canots. Comment celui-ci est-il original? Comment reflète-t-il votre personnalité ?»

Bien que la création de sous-groupes de travail et les échanges en grands groupes aient pu faciliter la mise en œuvre d'une dynamique collaborative, l'implication des élèves aurait pu se refléter aussi par l'articulation de questions tout au long de la progression du travail de création. Les échanges entre les différents groupes de travail auraient pu être plus abondants. Cette réflexion met en lumière l'une des limites de cette étude puisque l'analyse des interactions des groupes de travail ne tient pas compte de leur travail en atelier.

\section{Un appui timide d'artéfacts matériels}

À des fins de partage, mais aussi de rétention de l'information, une classe peut être davantage impliquée dans son apprentissage par la création systématique d'artéfacts de connaissances partagées tels que des plans, des dessins, des diagrammes et des idées externalisées (Bereiter, 2002; Bereiter et Scardamalia, 1993). De plus, c'est tout le processus de création de connaissances qui aurait pu être soutenu par la création d'artéfacts variés et l'exploitation d'illustrations pour appuyer les idées aurait pu être encouragée par des recherches plus soutenues sur Internet. La réalisation par les élèves d'une plus grande quantité de croquis aurait pu inciter les interactions et entamer le dialogue entre les groupes de travail. Enfin, le KF contient un outil de dessin qui aurait pu être exploité pendant la création.

La création d'une pensée artistique commune : de l'idée personnelle à l'idée collective

La pensée artistique a été relevée à travers le discours sur l'avancement de l'objet d'art. Les résultats montrent que les élèves ont développé cet objet en négociant seulement les termes des contraintes matérielles et personnelles/ expressives. Les considérations esthétiques n'ont donc pas été abordées lors de la création de cet objet. L'objet-classe a donc été établi à travers la matérialisation des éléments propres à leur culture. C'est à travers la négociation des objectifs et des contraintes de la proposition de création que les élèves ont travaillé leur objet d'équipe. Cette fois, les élèves ont surtout ajouté des composantes personnelles et des caractéristiques esthétiques. Selon les résultats de la grille des considérations artistiques, les élèves, à travers les énoncés réflexifs sur leur propre démarche, ont partagé en plus grande proportion leur expérience personnelle. Ces éléments de leur culture, évoqués ici, sont propres à l'individu et font référence à ce que les élèves connaissent : c'est-à-dire à leur goût, à leur intérêt et aux éléments liés à leur environnement. Chaque élève a ainsi partagé sa propre individualité, mais le caractère collaboratif du travail de création a permis aux élèves de créer des synthèses collectives de leurs idées également matérialisées dans leur œuvre. En d'autres mots, les différents concepts et significations ont été transformés dans leur produit créatif. De même, les élèves ont illustré leur propos en spécifiant de quelle manière les résultats des œuvres ont été des témoins de la spécificité de chacun. C'est ce qui nous porte à conclure que les élèves ont donc réalisé des œuvres personnelles témoignant de leur réalité commune.

\section{Pistes de recherche et conclusion}

À partir de certaines limites de la présente étude, nous proposons différentes pistes de recherches pouvant être prises en considération. Le travail de conception matérielle des élèves lors 
des activités en atelier n'a pas été l'objet d'une analyse. Seules les notes écrites sur le KF ont été utilisées. Effectivement, il aurait été intéressant d'analyser les enregistrements des conversations des différentes équipes de travail en atelier pendant la phase de création. Les enregistrements des conversations in situ et celles liées aux activités préparatoires à la création auraient pu grandement nous éclairer sur la dynamique collaborative de la classe en réseau et nous aurions pu davantage suivre ses transformations en cours de projet.

De plus, le projet en réseau De jeunes artistes sans frontières mis en œuvre dans cette étude a offert la possibilité à l'enseignante de réfléchir sur ses intentions pédagogiques et sur son transfert dans sa pratique. En effet, les enseignants qui participent à des discussions théoriques de leur domaine le font pour résoudre les problèmes liés à leur pratique d'enseignement, mais aussi à des fins d'innovation. Toutefois, cette forme de développement professionnel (Voogt et al., 2015) n'a pas été analysée en tant que telle. Il aurait été intéressant de suivre les réflexions pédagogiques de l'enseignante pendant la réalisation du projet à l'aide de la tenue d'un journal ou par des entrevues, par exemple.

Le présent devis a été réalisé avec la collaboration de l'enseignante, mais il inclut également des experts du milieu des arts, c'est-à-dire des artistes. Selon Kangas, SeitamaaHakkarainen et Hakkarainen, (2007), il est essentiel de fournir aux étudiants l'expérience d'interagir et de travailler avec un expert adulte. La collaboration avec l'artiste aurait aussi pu s'étendre à la phase de création, ce qui aurait favorisé le transfert de connaissances vers l'objet d'art. Ainsi, le travail réalisé en atelier avec l'artiste aurait pu être le sujet d'une analyse. Certaines insécurités des enseignants, liées à leur inexpérience en arts plastiques comme matière disciplinaire, pourraient être diminuées grâce à une telle collaboration.

En conclusion, puisque cette étude est une première dans le contexte québécois, voire nordaméricain, plusieurs pistes de recherche restent à explorer. Nous croyons qu'il faut stimuler la création d'artéfacts variés et ce, à toutes les étapes du processus de création afin de permettre aux élèves de s'engager davantage dans leur apprentissage. Il faut également encourager les interactions en misant sur le questionnement, la négociation des idées et une collaboration plus marquée avec un artiste. 


\section{Références}

Allaire, S. et Laferrière, T. (2013). Synthèse d'idées et de travaux à propos de la coélaboration/création de connaissances et du Knowledge Forum. Revue Adjectif http://www.adjectif.net/spip/spip.php?article234

Bereiter, C. (2002). Education and mind in the knowledge society. Lawrence Erlbaum.

Bereiter, C. et Scardamalia, M. (2014). Knowledge building and knowledge creation: One concept, two hills to climb. Dans S. Chee Tan et H. Jeong So, Knowledge creation in education (p. 35-52). Springer.

Chi, M. T. (1997). Quantifying qualitative analyses of verbal data: A practical guide. The journal of the learning sciences, 6(3), 271-315.

Engeström, Y. (1987). Learning by expanding. Orienta-Konsultit.

Gosselin, P. (2006). La recherche en pratique artistique : spécificité et paramètres pour le développement de méthodologies. Dans P. Gosselin et É. Le Coguiec, La recherche création. Pour une compréhension de la recherche en pratique artistique(p. 21 à 32). Presses de l’Université du Québec.

Hakkarainen, K. (2003). Emergence of progressive-inquiry culture in computer-supported collaborative learning. Learning Environments Research, 6(2), 199-220.

Hakkarainen, K. P., Palonen, T., Paavola, S. et Lehtinen, E. (2004). Communities of networked expertise: Professional and educational perspectives.

Hamel, C., Turcotte, S. et Laferrière, T. (2013). L'accompagnement d'une communauté d'apprentissage professionnelle en réseau au service du développement professionnel de ses membres. Éducation et francophonie, 41(2), 84-101.

Härkki, T., Seitamaa-Hakkarainen, P. et Hakkarainen, K. (2016). Material knowledge in collaborative designing and making-A case of wearable sea creatures. FORMakademisk.

Hennessy, S. et Murphy, P. (1999). The potential for collaborative problem solving in design and technology. International Journal of Technology and Design Education, 9(1), 1-36.

Kangas, K., Seitamaa-Hakkarainen, P. et Hakkarainen, K. (2007). The artifact project—History, science, and design inquiry in technology enhanced learning at elementary level. Research and Practice in Technology Enhanced Learning, 2(03), 213-237.

Kaptelinin, V. et Nardi, B. A. (2006). Acting with technology: Activity theory and interaction design. MIT press. 
Krippendorff, K. (2004). Reliability in content analysis. Human communication research, 30(3), 411-433.

Laferrière, T., Hamel, C., Laberge, C. et Allaire, S. (2005). Les communautés d'apprenants en réseau: un modèle intégrateur pour l'éducation à la citoyenneté au XXIe siècle. Enseigner et éduquer à la citoyenneté.

Lahti, H., Seitamaa-Hakkarainen, P., Kangas, K., Härkki, T. et Hakkarainen, K. (2016). Textile teacher students' collaborative design processes in a design studio setting. Art, Design \& Communication in Higher Education, 15(1), 35-54.

Lahti, H. et Seitamaa-Hakkarainen, P. (2005). Towards participatory design in craft and design education. CoDesign, 1(2), 103-117.

Lakkala, M. (2010). How to design educational settings to promote collaborative inquiry: Pedagogical infrastructures for technology-enhanced progressive inquiry [thèse de doctorat, Université d'Helsinki. Helsingin yliopisto.https://helda.helsinki.fi/bitstream/handle/10138/19887/howtodes.pdf?sequence=2\&isAl lowed $=\mathrm{y}$

Lakkala, M. Paavola, S. Kosonen, K. Muukkonen, H. Bauters, M. et Markkanen, H. (2009) Main functionalities of the Knowledge Practices Environment (KPE) affording knowledge creation practices in education. ISLS International Society of the Learning Sciences, 297-306. http://hdl.handle.net/10138/18654

Lamb, J. M. et Kallal, M. J. (1992). A conceptual framework for apparel design. Clothing and Textiles Research Journal, 10(2), 42-47.

Métivier, J. (2015). Le développement professionnel en réseau pour l'enrichissement de l'environnement d'apprentissage d'une classe de niveau élémentaire du Sénégal [mémoire de maîtrise]. Université Laval.Corpus.ulaval.ca. https://corpus.ulaval.ca/jspui/bitstream/20.500.11794/25909/1/31599.pdf

Nimkulrat, N., de Freitas, N., Pantouvaki, S. et Seitamaa-Hakkarainen, P. (2016). Experience, Materiality, Articulation. Studies in Material Thinking, 14.

Nonaka, I. et Takeuchi, H. (1995). The knowledge-creating company: How Japanese companies create the dynamics of innovation. Oxford university press.

Paavola, S., Engeström, R. et Hakkarainen, K. (2012). The trialogical approach as a new form of mediation. Collaborative knowledge creation, 1-14.

Paavola, S. et Hakkarainen, K. (2014). Trialogical approach for knowledge creation. Dans S. Chee Tan et H. Jeong So, Knowledge creation in education(p. 53-73). Springer. 
Paavola, S., Lipponen, L. et Hakkarainen, K. (2002). Epistemological foundations for CSCL: A comparison of three models of innovative knowledge communities. Dans G. Stahl, Computer Support for Collaborative Learning. Foundations for a CSCL Community.

Paavola, S., Lipponen, L. et Hakkarainen, K. (2004). Models of innovative knowledge communities and three metaphors of learning. Review of educational research, 74(4), 557-576. Québec. (2001). Programme de formation de l'école québécoise.

Scardamalia, M. et Bereiter, C. (1994). Computer support for knowledge-building communities. The journal of the learning sciences, 3(3), 265-283.

Seitamaa-Hakkarainen, P., Lahti, H. et Hakkarainen, K. (2005). Three design experiments for computer-supported collaborative design. Art, Design \& Communication in Higher Education, $4(2), 101-119$.

Seitamaa-Hakkarainen, P., Viilo, M. et Hakkarainen, K. (2010). Learning by collaborative designing: technology-enhanced knowledge practices. International Journal of Technology and Design Education, 20(2), 109-136.

Sfard, A. (1998). On two metaphors for learning and the dangers of choosing just one. Educational researcher, 27(2), 4-13.

Simard St-Pierre. (2016). Exploration du travail d'une classe en réseau sur un objet partagé en situation d'arts plastiques[mémoire de maîtrise]. Université Laval. Corpus.ulaval.ca. https://corpus.ulaval.ca/jspui/bitstream/20.500.11794/27219/1/32770.pdf

Souriau, É. et Souriau, A. (1999). Vocabulaire d'esthétique. Quadrige/PUF.

Tan, S. C. et Tan, Y. H. (2014). Perspectives of knowledge creation and implications for education. Dans S. Chee Tan et H. Jeong So, Knowledge creation in education (p. 11-34). Springer.

Voogt, J., Laferrière, T., Breuleux, A., Itow, R. C., Hickey, D. T. et McKenney, S. (2015). Collaborative design as a form of professional development. Instructional Science, 43(2), 259282.

Vygotsky, L. S. (1971). The psychology of art (Scripta Technica, Inc., Trans.).MIT press. (Original work published 1925).

\footnotetext{
${ }^{1}$ Le KF est une plateforme numérique de collaboration qui permet de soutenir les interactions entre les élèves lors de la réalisation d'activités, de projets ou d'investigations collectives. Des artéfacts d'apprentissage ou de connaissances sont donc créés.

${ }^{2}$ Allaire et Laferrière (2013) précisent que l'expression coélaboration de connaissances tend maintenant à être synonyme de l'expression création de connaissances.
} 
${ }^{3}$ Sfard (1998) propose deux métaphores qui permettent d'illustrer les différentes théories de l'apprentissage : celle de l'acquisition de la connaissance (AM) et celle de la participation à des fins d'apprentissage (PM).

${ }^{4}$ La création de connaissances comprend les théories de Nonaka et Takeuchi (1995) sur la création de connaissances en contexte organisationnel, la théorie de Bereiter (2002) et la coélaboration de connaissances, ainsi que la théorie de l'activité où s'entreprennent des cycles d'apprentissage expansif (Engeström, 1987).

${ }^{5}$ La démarche de création adaptée en réseau amène les élèves à travailler sur le KF afin de coélaborer leurs idées et leur choix, tandis que l'expérimentation avec des matériaux se vit en atelier. Cela étant dit, le processus de création s'est vécu de manière à matérialiser les idées par des allers-retours entre l'atelier et le KF.

${ }^{6} \mathrm{Ce}$ plugiciel du KF permet d'extraire les enfilades des contributions du discours écrit. 